Nota de investigación

\title{
Situación y desafíos de la producción y comercialización de maíz en Tulantepec, Hidalgo
}

\begin{abstract}
Alma Janeth Aguilar-Curiel ${ }^{1}$
Juan Manuel Quintero-Ramírez ${ }^{2 \S}$

Dora Ma. Sangerman-Jarquín ${ }^{3}$

José Miguel Omaña Silvestre ${ }^{4}$

${ }^{1}$ Universidad Politécnica Metropolitana de Hidalgo. Ex Hacienda San Javier, Tolcayuca núm. 1009, Tolcayuca, Hidalgo. CP. 43860. (jancurie1411@gmail.com). ${ }^{2}$ Consejo Nacional de Ciencia y Tecnología. Av. Insurgentes Sur 1582, Col. Crédito Constructor, Alcaldía Benito Juárez, Ciudad de México. CP. 03940. (juan.quintero@conacyt.mx). ${ }^{3}$ Campo Experimental Valle de México-INIFAP. Carretera Los ReyesTexcoco km 13.5, Coatlinchán, Texcoco, Estado de México. CP. 56250. Tel. 55 38718700, ext. 85353 (sangerman.dora@inifap.gob.mx). ${ }^{4}$ Colegio de Posgraduados. Carretera México-Texcoco km 36.5, Montecillo, Texcoco, Estado de México. CP. 56230. (miguelom@ colpos.mx).

$\S^{\S}$ Autor para correspondencia: quintauro82@gmail.com.
\end{abstract}

\section{Resumen}

El municipio de Santiago Tulantepec de Lugo Guerrero, Hidalgo, se ve favorecido en la producción de maíz en sus distintas variedades debido a que las condiciones climatológicas y el tipo de suelo se prestan para producir este cultivo. El objetivo de esta investigación fue analizar la relación entre la producción y comercialización de maíz, para conocer los beneficios económicos que resulte en mayor utilidad para los productores de la zona. El análisis demandó conocer su producción y comercialización y los costos de siembra hasta su cosecha, tomando información en el ciclo productivo 2017-2018. La recopilación de datos se basó en una muestra finita; a través, de un muestreo aleatorio simple, con un instrumento de recopilación de datos de dieciocho ítems, aplicado a productores de maíz, tomando como universo a los 407 productores inscritos en el programa ProAgro, calculando y resultando la aplicación a 198 como muestra para el estudio. Se aplicó una prueba de confiabilidad mediante Alfa de Crobach, la cual arrojó 0.702, para dar confiabilidad y validez a la investigación. La información recopilada se procesó en una base de datos del programa estadístico SPSS, obteniendo las medidas de tendencia central. Finalmente, a partir de los resultados se hacen recomendaciones y propuestas basadas en la mejora de los procedimientos de comercialización para los productores, que puede dar pauta a nuevas investigaciones para el sector agrario en el municipio.

Palabras clave: maíz, producción y comercialización, Tulantepec.

Recibido: septiembre de 2021

Aceptado: octubre de 2021 
La importancia del maíz (Zea Mays L.) en México surge a través de la estructura histórica del patrón alimentario ya que el maíz tiene un peso determinante en el consumo de la población. De acuerdo con datos de la Organización de las Naciones Unidas para la Alimentación y la Agricultura (Food and Agriculture Organizatión, en sus palabras originales), 47\% de la demanda total de maíz en México es utilizado para consumo humano directo, $32 \%$ es para forraje, $5 \%$ para usos industriales y el resto en otros usos (FA0 2018).

El municipio de Santiago Tulantepec de Lugo Guerrero, ubicado en el estado Hidalgo, se conforma por 21 colonias y localidades, que son favorecidas en la producción de maíz en sus distintas variedades debido a que las condiciones climatológicas y el tipo de suelo de la región, son idóneas para el cultivo de maíz.

La importancia del sector agropecuario para el estado de Hidalgo radica en la contribución del producto interno bruto (PIB) estatal, que en promedio es de 9\%, así como por la generación de empleo ya que $23.5 \%$ de la población económicamente activa trabaja en alguna actividad relacionada con este sector y $62.8 \%$ de la población vive en zonas rurales (SAGARPA, 2011). En materia de competitividad, el estado ocupa el lugar $22^{\circ}$ de los estados de la República Mexicana (IMCO, 2018). La problemática que se aborda en esta investigación recae en analizar la producción y comercialización de maíz en el municipio de Santiago Tulantepec de Lugo Guerrero, Hidalgo, para el ciclo productivo 2017-2018, con el propósito de conocer el beneficio económico que resulta de la actividad productiva.

El objetivo que se persigue es la relación que existe entre la producción y la comercialización de maíz, con base en el estudio de un ciclo productivo, con la finalidad de conocer el beneficio económico de la actividad productiva. Con esto, el tener una existencia de una relación entre la producción y la comercialización de maíz que afecta el impulso del comercio internacional en el municipio de Santiago Tulantepec de Lugo Guerrero; como la hipótesis de esta investigación.

Considerando el alto potencial que tiene este sector en el municipio, la necesidad de mantener e incrementar su crecimiento productivo, el impacto potencial en beneficios económicos y sociales para la región y la oportunidad que se presenta para convertirse en un sector competitivo, es necesario un estudio que pueda determinar los parámetros de rentabilidad en la producción y comercialización del maíz, permitiendo brindar la posibilidad a los productores del municipio contar con un estudio que manifieste los beneficios derivados de la producción y comercialización de maíz, considerando que las condiciones exigidas para la adaptación y desarrollo de la planta, son básicamente con las que cuenta la región, donde la producción de maíz podría ser una opción de fuente de ingreso para los productores.

Se buscó identificar con base al estudio de la producción y comercialización de maíz, las posibilidades de aprovechar el maíz como alternativa que permita guiar el desarrollo de esta región, planteando soluciones viables económicas y sociales ligados a los problemas que atañen en el municipio de Santiago Tulantepec, Hidalgo.

El municipio está localizado a una distancia de $51 \mathrm{~km}$ de Pachuca la capital del estado de Hidalgo. Se encuentra a una altura sobre el nivel del mar de $2180 \mathrm{~m}$ y sus coordenadas geográficas extremas son latitud norte $20^{\circ} 02^{\prime} 21^{\prime \prime}$ respecto al meridano de Greenwich. Cuenta con una extensión territorial de $90 \mathrm{~km}^{2}$ y representa $0.37 \%$ de la superficie total del estado de Hidalgo. 
El tipo de maíz cultivado en este municipio es el maíz forrajero en modalidad de riego para la temporada de primavera-verano (PV). Para el maíz blanco se cuenta con riego y de temporal ambos en modalidad PV, de las cuales, de riego se sembraron y cosecharon 252 ha, mientras que de modalidad de temporal el maíz blanco se sembró y cosecho en 1653 ha con un volumen de producción de $2349.5 \mathrm{t}$.

Considerando el alto potencial que tiene este sector en el municipio de Santiago Tulantepec de Lugo Guerrero la necesidad de mantener e incrementar su crecimiento productivo, el impacto potencial en beneficios económicos y sociales para la región y la oportunidad que se presenta para que se convierta en un sector competitivo, se es necesaria una investigación que determine los parámetros de rentabilidad en la producción y comercialización del maíz, que brinde la posibilidad a los productores del municipio contar con un estudio que manifieste los beneficios derivados de la producción y comercialización de maíz, considerando que las condiciones exigidas para la adaptación y desarrollo de la planta son básicamente con las que cuenta la región, donde la producción de maíz podría ser una opción a examinar como fuente de ingreso para los productores.

La investigación se realizó mediante el trabajo de campo o estudio en las parcelas con los productores de maíz en Santiago Tulantepec, Hidalgo, lo que permite obtener los datos necesarios para conocer las labores culturales y agrícolas que se deben aplicar durante un ciclo productivo, así como las actividades realizadas en el proceso de comercialización del maíz, para identificar los beneficios que se obtienen de la producción y comercialización de este producto.

Los datos están fundamentados en un diseño no experimental, de estudio de campo trasversal ya que la aplicación de la encuesta se hizo en un solo momento a través de un cuestionario con consiste en respuesta de opción múltiple y la asignación de datos que los mismos productores respondían, como son los costos que tienen para producir el maíz en la temporada.

El objeto de estudio son los productores de maíz que se encuentran inscritos en el Programa ProAgro registrada por la SAGARPA, exclusivos de las localidades del municipio de Santiago Tulantepec, a quienes se les aplico un cuestionario para recoger datos que permiten el análisis de las variables a estudiar y con ello la comprobación de la hipótesis. De acuerdo con los datos proporcionados por la dirección de desarrollo rural en el área administrativa del municipio se muestra que los productores registrados dedicados a la producción de maíz son 407 (PROAGRO, 2018).

Para la obtención de la muestra, se desea conocer cuántos cuestionarios se tendrían que aplicar a esta población de productores de la zona, determinándose bajo la siguiente ecuación: $\mathrm{N}=\frac{\mathrm{NZ}^{2} \mathrm{pq}}{\mathrm{e}^{2}(\mathrm{~N}-1)+\left(\mathrm{Z}^{2} \mathrm{pq}\right)}$; donde: $\mathrm{N}=$ tamaño de la población o universo; e = valor entre $1 \%(0.01)$ y $9 \%$ (0.09), se eligió $5 \% ; p=$ cuando no se conoce generalmente se la da el valor de $0.5 ; q=1-p$.; $Z=$ constante que depende del nivel de confianza que asignemos y corresponde a una distribución Normal o de Gauss, con un nivel de confianza de 0.95, se utiliza 1.96.

Los datos integrados en la fórmula son los siguientes: $\mathrm{n}=$ $\frac{(407)(1.96)^{2}(0.5)(0.5)}{(0.05)^{2}(407-1)+(1.96)^{2}(0.5)(0.5)}=197.88 \rightarrow 198$ encuestas. El resultado es de 197.88 cuestionarios, pero por cuestiones de redondeo y determinación en la investigación se realizarán 198 cuestionarios. 
Para la recopilación de información de campo, las encuestas se aplicaron de manera directa, para ello se acudió directamente con los productores seleccionados en la muestra del municipio de Santiago Tulantepec, explicando el motivo de la investigación y los criterios para responder a cada una de las preguntas que se realizaban.

Aunque existen fuentes de información primaria y secundaria, este estudio está basado en el uso de fuentes de información primarias. Además, la investigación está apoyada del uso de tecnologías de la información para la obtención de datos y documentos digitales. Las fuentes de información primarias a utilizar en la investigación son mediante la aplicación de encuestas, para la obtención de la información sobre los costos en el proceso de producción, los volúmenes y la comercialización realizada por los productores de maíz en Santiago Tulantepec.

La recopilación de la información inicio con una prueba piloto para revisar si las preguntas estaban bien estructuradas y planteadas haciéndose a cabo en un día con 12 productores de maíz en el municipio de Santiago Tulantepec para determinar la confiablidad del instrumento hasta obtener el nivel alcanzable en el Alfa de Crombach (0.702 de fiabilidad), corrigiendo los errores $\mathrm{u}$ observaciones que se encontraron. Después se procedió a hacer las encuestas dentro de en un horario de 10 de la mañana a 6 de la tarde, donde se aplicaron el total de los instrumentos con 18 ítems.

Las 198 encuestas se realizaron en casa de algunos productores y algunos que se encontraban en el campo haciendo sus labores. Después de aplicar las muestras se ordenan los folios de los instrumentos para su captura y procesamiento. Se elaboró un instrumento impreso, el cual se divide en 4 partes la primera sección se refiere a sus datos generales del productor; la segunda sección basada en 8 preguntas donde se indaga información de la producción para maíz, la tercera sección hace siete preguntas referentes a la comercialización del maíz que realizan, del cual, si no se tenía, se pasaba a la siguiente sección; y la última sección se cuestiona con 3 preguntas y el llenado de una tabla de los costos que tienen al realizar las diferentes actividades agrícolas para producción de maíz en sus tierras.

Al terminar la aplicación de los instrumentos para obtención de información, se ocupó el programa estadístico informático SPSS Statistics ${ }^{\circledR}$ donde se generan las gráficas para la evaluación de los resultados de las encuestas y facilitar la interpretación de las encuestas. Dentro de los resultados que arroja el programa da las frecuencias de cada respuesta lo que facilita la interpretación de los resultados de cada pregunta realizada.

La presente investigación permitió cumplir el objetivo general, que consistió en realizar un análisis de la producción y comercialización de maíz en el municipio de Santiago Tulantepec, Hidalgo, con la finalidad de identificar algunos puntos a fortalecer en los procesos de producción y comercialización y así diseñar una propuesta que impulse la generación de empleo y beneficios y utilidades hacia los productores de este municipio (ASERCA, 2018).

De esta investigación, se puede concluir diferentes aspectos importantes, lo que arrojándonos en las encuestas se inicia que, generalmente las personas que aún se dedican a la siembra de maíz son de edades mayores, en promedio oscilando arriba de los 50 años. Son productores que conocen el quehacer y están acostumbrados a las labores de campo y en conjunto se reporta que los productores tienen estudios máximos de primaria $(85.86 \%)$, lo que quiere decir que hay un alto grado de analfabetismo entre ellos (CIMMYT, 2008; FAOSTAT, 2018). 
Por el gran número que se encontró de productores en el municipio de Santiago Tulantepec de Lugo Guerrero, Hidalgo se puede teorizar que el área cuenta con tierra fértil, el clima es idóneo y que se presta a las condiciones para obtener este cultivo que es de suma importancia para el consumo humano. El maíz que se cosecha en los predios y parcelas de este municipio se siembra en diversas variedades como lo son, el maíz blanco (9.6\%), maíz amarillo (3.03\%), maíz negro (1.52\%), maíz hibrido y otras variedades nativas (85.86\%) (Latham, 2002; USDA, 2018).

La gran mayoría de productores (89.9\%) tiene predio de 1 a 5 ha para el cultivo, mientras que solo $1.52 \%$ tiene más de 15 hectáreas Rudiño (2011). Los años que tienen de estar sembrando y cosechando su maíz en $88.38 \%$ de productores es mayor a 15 años realizando esta actividad, $7.07 \%$ va de 11 a 15 años, $2.53 \%$ tienen entre 6 y 10 años sembrando y solo $2.02 \%$ tienen entre 1 a 5 años con estas actividades agrícolas de maíz.

Los productores en su mayoría siembran por costumbre y propia ocupación y no buscan obtener un beneficio por la venta de sus cosechas más solo lo utilizan para autoconsumo, debido a que los gastos de producción son muy elevados y las utilidades muy pocas. En cuanto a las labores de campo, de la totalidad de cultivos, solo $0.51 \%$ es de riego, mientras que el resto es por temporal con las lluvias de primavera-verano como refiere Lesur (2005). Así también, 42.93\% realiza la fertilización y abono de las tierras con diferentes materias orgánicas para nutrir la tierra.

En la ocupación de personal como jornal para realizar las diferentes actividades, la mayoría de los productores (79.29\%) tienen un gasto de entre $\$ 200.00$ a $\$ 300.00,19.7 \%$ paga entre $\$ 100.00$ a $\$ 200.00$ y con un pago entre $\$ 300.00$ a $\$ 400.00$ son el $0.51 \%$ de productores INEGI (2018). Para el desembolso de estos jornales, los productores lo hacen de sus propios ingresos, pues solo $4.04 \%$ cuenta, con algún apoyo gubernamental destinado al campo, mientras que $95.96 \%$ no cuenta con otro ingreso para estas labores

En cuanto a la maquinaria, equipos e implementos agrícolas, $49.49 \%$ realiza sus actividades agrícolas con el tractor, mientras que el resto lo sigue haciendo con yunta de animales de esto, $40.91 \%$ tiene la maquinaria o equipo de producción propio, $58.08 \%$ lo rentan y $0.51 \%$ alguna persona se los presta para realizar su labor. Con estas actividades, $27.27 \%$ aun realiza las podas y deshierbe en sus terrenos para el cuidado de malezas y hierbas (Sampieri et al., 2010).

Con respecto a la variable de comercialización, es donde se presenta más debilidad, ya que el sector tiene poca o nula experiencia en el mercado y desconocen los procesos para exportar e incluso, ignoran los procesos para comerciar en mercados locales (Varela et al., 2010). La variable de producción también presenta debilidad debido a que los productores no cuentas con herramienta que les podría beneficiar en el momento de realizar sus procesos y así reducir tiempo y costos SAGARPA (2018). Entre estos procesos esta la maquinaria de los productores, la mayoría no cuenta con equipo propio y esto eleva sus costos al solicitar que les realicen las labores.

\section{Conclusiones}

Se cuenta con un gran número de productores; sin embargo, carecen de una cultura empresarial, en planeación y organización, así como de cultura financiera. Para elevar el nivel de competitividad de los productores es recomendable se organicen y desarrollen habilidades directivas, desarrollar capacidades financieras, fortalecer el trabajo en equipo y la capacidad de adaptarse a los cambios. 
Es importante que entes gubernamentales o privados, realicen capacitación para que los productores puedan organizarse y desarrollarse. Se puede crea una sociedad de productores donde se les apoye con conocimientos de comercialización y aplicación de la logística y puedan obtener mayor beneficio de sus cultivos por medio de la creación de un centro de acopio en donde se concentre toda la producción de los pequeños productores de maíz; así como, se observó que la mayoría de los productores son de edad avanzada.

Así también, se puede buscar apoyos gubernamentales para la obtención del tractor e implementos, dando aportaciones entre gobierno y productores para trabajar los terrenos sin realizar más gasto del que realizan hoy.

Otro aspecto que aportaría valor como sociedad de productores es la certificación de su producto con estándares de calidad y así comerciar a precios competitivos. Así como, conseguir un seguro a las cosechas para que en el caso de que las condiciones climatológicas afecten la producción, éstas no se pierdan en lo parcial o su totalidad y los productores no se vean afectados en su economía.

\section{Literatura citada}

ASERCA. 2018. Gobierno de México. Maíz grano cultivo representativo de México. https://www.gob.mx/aserca/articulos/maiz-grano-cultivo-representativo-de-mexico?idiom=es.

CIMMYT. 2008. Centro de Investigación para Mejoramiento de Maíz y Trigo. https://www.cimmyt.org/es/?s=maiz.

FAOSTAT. 2018. Food and Agriculture Organization of the United States. Top 10 de producción en México 2018. http://www.fao.org/faostat/en/\#data/QC/visualize.

FAO. 2018. Food and Agriculture Organization. http://www.fao.org/home/es/.

IMCO. 2018. Instituto Mexicano para la Competitividad. Índice de competitividad estatal. http://imco.org.mx/indices/el-estado-los-estados-y-la-gente/resultados/entidad/13-hidalgo.

INEGI. 2018. Instituto Nacional de Estadística y Geografía. Cifras de Hidalgo. https://www.inegi.org.mx/app/areasgeograficas/?ag=13.

Latham, M. 2002. Nutrición humana en el mundo en desarrollo. Colección FAO: Alimentación y nutrición núm. 29. Organización de las Naciones Unidas para la Agricultura y la Alimentación. http://www.fao.org/3/w0073s/w0073s00.htm\#Contents.

Lesur, L. M. 2005. Manual de cultivo de maíz, una guía paso a paso. Ed. Trillas. México, DF. 18$37 \mathrm{pp}$.

Rudiño, L. 2011. Iniciativas para elevar el rendimiento del maíz de temporal: metodología exitosa generada por campesinos. La Jornada del Campo (21).

SAGARPA. 2011. Resultados del estudio de diagnóstico sectorial en el estado de Hidalgo 2010. https://www.academia.edu/27442118/informe_final_diagnostico_sectorial_estado_de_hidalgo.

SAGARPA. 2017. Planeación agrícola nacional 2017-2030. Secretaria de Agricultura Ganadería, Desarrollo Rural, Pesca y Alimentación. https://www.gob.mx/agricultura/acciones-yprogramas/planeacion-agricola-nacional-2017-2030-126813.

Sampieri, R. H.; Collado, C. F. y Lucio, P. B. 2010. metodología de la investigación 5ª (Ed.). Mc Graw Hill. México, DF. 4-10 pp.

USDA. 2018. United States Department of Agriculture. https://www.nass.usda.gov/charts_and_ maps/crops_county/cr-pr.php.

Varela, A. G. 2010. Competitividad y ventajas comparativas de la producción de maíz en México. Rev. Mex. Cienc. Agríc. 1(3):381-396. 